\title{
Protective effect of Rhizoma drynariae extract on osteoporosis in ovariectomized rat model
}

\author{
Yu-li Cai ${ }^{1 *}$ and Xing-cai Zhang ${ }^{2}$ \\ ${ }^{1}$ Department of Orthopedics, ${ }^{2}$ Internal Medicine, The Affiliated Hospital of Shandong University of Traditional Chinese Medicine, \\ Jinan, 250011, Shandong Province, China \\ *For correspondence: Email: caiyuli133494@163.com; Tel: +86053168616666
}

\begin{abstract}
Purpose: To investigate the therapeutic effect of Rhizoma Drynariae extract (RDE) on ovariectomyinduced osteoporosis in rats.

Methods: Female Sprague-Dawley rats were randomly assigned to a sham-operated group (control) and five ovariectomy (OVX) subgroups: OVX with vehicle (OVX), OVX with 17ß-estradiol ( $E_{2}, 25$ $\mu \mathrm{g} / \mathrm{kg} /$ day), and OVX with RDE doses (40, 80, and $160 \mathrm{mg} / \mathrm{kg} /$ day). Daily oral administration of E2 or $R D E$ started 4 weeks after OVX and lasted for 16 weeks. The bone mineral density (BMD) of the L4 vertebrae and right femurs was estimated. The length of each femur was measured with a micrometer gauge, and the center of the diaphysis determined. Three representatives $L 4$ vertebrae were selected to evaluate the trabecular microarchitecture. Serum alkaline phosphatase $(A L P)$, urinary calcium (U-Ca), urinary phosphorus (U-P), urinary creatinine (Cr) and osteocalcin (OC) levels were measured.

Results: The study showed that high-dose of RDE significantly inhibited the bone mineral density (BMD) reduction of $L 4$ vertebrae $\left(0.20 \pm 0.02 \mathrm{~g} / \mathrm{cm}^{3}, p<0.05\right)$ and femurs $\left(0.18 \pm 0.02 \mathrm{~g} / \mathrm{cm}^{3}, p<0.05\right)$ caused by OVX and prevented the deterioration of trabecular microarchitecture $(p<0.05)$, which were accompanied by a significant decrease in skeletal remodeling $(p<0.05)$ as evidenced by the lower levels of bone turnover markers. High-dose of RDE improved morphometric parameters, namely, $T b-N$ (3.8 $\pm 0.2 \mathrm{~mm}, p<0.05)$, Tb-Th $(0.083 \pm 0.011 \mathrm{~mm}, p<0.05)$ and Tb-Sp $(0.19 \pm 0.01 \mathrm{~mm}, p<0.05)$ in L4 vertebrae significantly. The present study indicates that the administration of RDE at higher doses over a 16-week period can prevent OVX-induced osteoporosis in rats without hyperplastic effects on the uterus.

Conclusion: Thus, RDE is a potential natural alternative for postmenopausal osteoporosis treatment in elderly women.
\end{abstract}

Keywords: Rhizoma Drynariae, Postmenopausal osteoporosis, Ovariectomy, Bone mineral density, Morphometric

Tropical Journal of Pharmaceutical Research is indexed by Science Citation Index (SciSearch), Scopus, International Pharmaceutical Abstract, Chemical Abstracts, Embase, Index Copernicus, EBSCO, African Index Medicus, JournalSeek, Journal Citation Reports/Science Edition, Directory of Open Access Journals (DOAJ), African Journal Online, Bioline International, Open-J-Gate and Pharmacy Abstracts

\section{INTRODUCTION}

Osteoporosis is a systemic skeletal disease characterized by reduced bone mass and microarchitectural deterioration of bone tissue with a consequent increase in bone fragility and susceptibility to fractures [1]. According to data released by the World Health Organization
(WHO), osteoporosis affects approximately million people throughout Europe, the USA, and Japan [2]. The incidence of osteoporosis increases dramatically with life expectancy. Accordingly, the risk of osteoporotic fractures and their associated costs is rising rapidly due to population aging [3]. In the elderly, hip fractures are closely associated with mortality [4]. 
Hormone deficiency is known to impair cancellous metaphyseal bone and reduce BMD in humans and animals; therefore, estrogen deficiency in post-menopausal women has been regarded as a critical cause of this population's susceptibility to osteoporosis [5]. Osteoporosis is twice as common in women as in men [6], and approximately one in three women over 50 years old experiences an osteoporotic fracture in her life time [7].

Clinically, hormone replacement therapy (HRT) has been a popular therapeutic strategy designed for postmenopausal osteoporosis [8,9]. However, the long-term application of HRT has potential malignant effects on reproductive tissues [10-13]. Other medicines that stimulate bone formation (e.g., growth hormone, sodium uoride, and parathyroid hormone) or inhibit bone resorption (e.g., bisphosphonates and calcitonin) may prevent bone loss progression in established osteoporosis. However, these medications are not effective for a large proportion of the world population, especially in developing countries, and these drugs have side effects, such as gastrointestinal reactions, cancers, osteonecrosis of the jaw, and reduced skeletal strength $[14,15]$. Consequently, to substitute or reduce the medicines used currently, there are efforts to develop new drugs with improved therapeutic efficacy, fewer undesirable side effects, and lower price.

$R$. drynariae, the dried rhizome of Drynaria fortune (Kunze) J.Sm. (Gu-Sui-Bu in Chinese), has been widely used as a kidney-tonifying and anti-osteoporosis herb for the treatment of nephrasthenia syndrome [16], osteoporosis $[17,18]$ and bone fracture [19] for thousands of years in China. Therefore, the aim of the present study was to systematically evaluate the effect of RDE on osteoporosis induced by OVX in rats.

\section{EXPERIMENTAL}

\section{Preparation of Rhizoma Drynariae extract}

The herbal samples of Rhizoma Drynariae were collected from Bozhou City, Anhui Province in China in July 2014. Taxonomic identification of the plant was performed by Professor Kang Hu of Shandong University of Traditional Chinese Medicine, in China. A voucher specimen (no. RDE 201409015) was deposited in Shandong University of Traditional Chinese Medicine, China for future reference.

One batch of herbal samples Rhizoma drynariae was dried in an oven. Aqueous extract of RDE was obtained by steeping the dried Rhizoma drynariae in water at $60{ }^{\circ} \mathrm{C}$ three times for $1 \mathrm{~h}$ each. Then drying the extracted fluid in an oven and freeze-drying the last extract. The same batch was extracted for 3 times. One gram powder was equivalent to about $1.5 \mathrm{~g}$ crude samples. The yield was $66.67 \%$.

\section{Animals and treatment}

Healthy three-month-old female Sprague-Dawley rats (weighing $220 \pm 10 \mathrm{~g}$ ). Were provided by the Experimental Animal Center of Shandong Province (Certificate no. SYXK 2004-0003). The animals had free access to feed and water, and were allowed to acclimatize for at least one week before use. The rat experiment was approved by the Animal Care and Use Committee of Shandong University of Traditional Chinese Medicine (approval ref no. 20120804) and was carried out in compliance with the Directive 2010/63/EU on the handling of animals used for scientific purposes [20].

60 rats were randomly divided into six groups of ten individuals: a sham-operated group (control) and five ovariectomy (OVX) subgroups, that is, OVX with vehicle (OVX), OVX with 17ß-estradiol (E2, $25 \mathrm{mg} / \mathrm{kg} /$ day), and OVX with RDE doses (40, 80 and $160 \mathrm{mg} / \mathrm{kg} /$ day $)$. Daily oral administration of $\mathrm{E}_{2}$ or RDE started 4 weeks after OVX and lasted for 16 weeks.

\section{Bone mineral density measurement}

The BMD of the L4 vertebrae and right femurs was estimated using dual-energy $x$-ray absorptiometry scanning (DEXA, GE Healthcare, USA) with small animal measurement. The measurements were expressed as grams of mineral contents per $\mathrm{cm}^{2}$ of surface area. Scans were performed by the same blinded technician.

\section{Three-point bending test}

Before mechanical testing, the rats were sacrificed by cervical spondylosis. Then the left femurs were slowly thawed at room temperature. The length of each femur (distance from the intermalleolar to the intercondylar region) was measured with a micrometer, and the center of the diaphysis was determined.

\section{Micro-CT analysis}

Based on the BMD, three representatives L4 vertebrae from each group were selected to evaluate the trabecular microarchitecture using eXplore Locus SP preclinical specimen microcomputed tomography (MicroCT, GE Healthcare, USA). Before the scans, the bones 
were positioned with gauze in the sample holder and allowed to reach room temperature. The L4 vertebrae were scanned from the anterior endplate in the posterior endplate direction (22 um/slice). The isotropic voxel resolution of bone was $22 \mathrm{um}^{3}$. The volume of interest (VOI) was selected as a region 25 slices away from the anterior endplate to the posterior endplate, ranging to 125 slices. The three-dimensional images were reconstructed with the purpose of visualization and display. After analyzing the VOI, morphometric bone parameters, including trabecular number ( $\mathrm{Tb}-\mathrm{N})$, trabecular separation (Tb-Sp), trabecular thickness (Tb-Th) were obtained. The VOI analysis was performed blindly by the same operator.

\section{Biochemical analysis of serum and urine specimens}

The serum of rats were collected by picking eyeball, and the urine of rats were collected by stimulating back. The levels of serum alkaline phosphatase (ALP), urinary calcium (U-Ca), urinary phosphorus (U-P), and urinary creatinine $(\mathrm{Cr})$ were measured on an automatic analyzer (Ciba-Corning 550, USA) using a diagnostic reagent kit. Serum osteocalcin (OC) concentration was determined using a rat $\mathrm{OC}$ ELISA kit (SanClemente, CA, USA).

\section{Statistical analysis}

The data are expressed as the mean \pm SD. Statistical analysis was performed using one-way ANOVA combined with Bonferroni's multiple comparison test using SPSS 16.0. Differences were considered statistically significant at $p<$ 0.05 .

\section{RESULTS}

\section{BMD of L4 vertebrae and femur}

The BMD of the $L 4$ vertebrae and femurs is presented in Table 1. These results demonstrate that OVX significantly decreased the BMD in the L4 vertebrae and femurs compared to the sham group ( $p<0.05)$. Compared to the OVX group, RDE treatment significantly prevented the BMD decrease in OVX-induced L4 vertebrae and femurs (all $p<0.05$ ) in a dose-dependent manner. $E_{2}$ also significantly increased the BMD of the $L 4$ vertebrae and femurs (both $p<0.05$ ), which was similar to that observed in the H-RDE group $(p>0.05)$.

\section{Mechanical testing of femur}

The results of the femur mechanical testing are presented in Table 2. Compared with the sham group, 16 weeks of estrogen deficiency significantly decreased the maximum load and maximum stress (both $p<0.05$ ). Meanwhile, higher dosage of RDE treatments (80 or 160 $\mathrm{mg} / \mathrm{kg} /$ day) markedly prevented the OVXinduced tendency to decrease these parameters $(p<0.05)$. $\mathrm{E}_{2}$ also increased these biomechanical parameters, which were significantly higher than those of the OVX group $(p<0.05)$. It is worth noting that the increase in maximum load observed for the H-RDE group was similar to that of $\mathrm{E}_{2}(p>0.05)$.

Table 1: Effect of RDE on bone mineral density (BMD) of L4 vertebrae and femur $(n=10)$

\begin{tabular}{|c|c|c|c|}
\hline Group & Dosage (mg/kg) & BMD of vertebrae $\left(\mathrm{g} / \mathrm{cm}^{3}\right)$ & BMD of femurs $\left(\mathrm{g} / \mathrm{cm}^{3}\right)$ \\
\hline Control & - & $0.22 \pm 0.03$ & $0.19 \pm 0.03$ \\
\hline OVX & - & $0.16 \pm 0.04$ & $0.14 \pm 0.04$ \\
\hline $\mathrm{E}_{2}$ & 0.025 & $0.20 \pm 0.03^{\pi}$ & $0.18 \pm 0.03^{\pi}$ \\
\hline L-RDE & 40 & $0.18 \pm 0.02^{\pi}$ & $0.16 \pm 0.02^{\pi}$ \\
\hline M-RDE & 80 & $0.19 \pm 0.02^{\pi}$ & $0.17 \pm 0.03^{\pi}$ \\
\hline $\mathrm{H}-\mathrm{RDE}$ & 160 & $0.20 \pm 0.02$ & $0.18 \pm 0.02$ \\
\hline
\end{tabular}

Table 2: Effect of RDE on femur mechanical properties $(n=10)$

\begin{tabular}{|c|c|c|c|}
\hline Group & Dosage $(\mathrm{mg} / \mathrm{kg})$ & Maximum load (N) & Maximum stress (MPa) \\
\hline Control & - & $124.7 \pm 5.6$ & $198.4 \pm 6.3$ \\
\hline ovX & _ & $925+4.8$ & $154.2+5.8$ \\
\hline$E_{2}$ & 0.025 & $116.3 \pm 5.2$ & $183.6 \pm 5.1$ \\
\hline L-RDE & 40 & $95.1 \pm 4.9$ & $157.2 \pm 4.9$ \\
\hline M-RDE & 80 & $105.3 \pm 5.5^{\pi}$ & $172.3 \pm 5.3^{\pi}$ \\
\hline H-RDE & 160 & $112.4 \pm 5.1^{\pi}$ & $178.6 \pm 5.5^{\star}$ \\
\hline
\end{tabular}

${ }^{*} P<0.05$ and ${ }^{* *} p<0.01$ versus OVX group.L-RDE: low dose of RDE, M-RDE: middle dose of RDE, H-RDE: high dose of RDE 


\section{Micro-CT data for L4 vertebrae}

Analysis of the representative samples (Table 3) indicated that OVX resulted in the deterioration of the trabecular bone microarchitecture, as demonstrated by the reduced Tb-N and Tb-Th compared with the sham group (both $p<0.05$ ). In contrast, Tb-Sp was significantly increased in response to OVX compared to the sham group ( $p$ $<0.05$ ). Higher dosage of RDE treatment (80 or $160 \mathrm{mg} / \mathrm{kg} / \mathrm{day}$ ) significantly improved the microarchitecture deterioration mentioned above $(p<0.05)$, while $E_{2}$ also reversed these parameters to the similar degree as that in the $\mathrm{H}$ RDE group $(p>0.05)$.

\section{Biochemical profile of serum and urine specimens}

Data presented in Table 4 show the effect of RDE on biochemical parameters in the serum and urine of OVX rats. Serum U-Ca/Cr, U-P/Cr, $A L P$, and $O C$ levels were significantly increased in the OVX group compared to the sham group $(p<0.05)$. All three RDE doses significantly decreased the $\mathrm{U}-\mathrm{Ca} / \mathrm{Cr}$ and ALP levels $(p<0.05)$ in a dose-dependent manner. Higher dosage of RDE treatment ( 80 or $160 \mathrm{mg} / \mathrm{kg} /$ day) decreased the U-P/Cr and OC levels $(p<0.05)$. Again, $\mathrm{E}_{2}$ administration also reversed these increases which were statistically significant. It is worth noting that the reductions in U-P/Cr, ALP, and $\mathrm{OC}$ in the H-RDE group were similar to those observed for $\mathrm{E}_{2}$ (all $p>0.05$ ).

Table 3: Effect of RDE on morphometric parameters in L4 vertebrae $(n=10)$

\begin{tabular}{|c|c|c|c|c|}
\hline Group & Dosage (mg/kg) & Tb-N (1/mm) & Tb-Th (mm) & Tb-Sp (mm) \\
\hline Control & - & $4.5 \pm 0.3^{\pi}$ & $0.097 \pm 0.014$ & $0.14 \pm 0.01$ \\
\hline OVX & - & $2.5 \pm 0.4$ & $0.065 \pm 0.012$ & $0.33 \pm 0.03$ \\
\hline $\mathrm{E}_{2}$ & 0.025 & $4.1 \pm 0.4$ & $0.088 \pm 0.013$ & $0.18 \pm 0.01$ \\
\hline L-RDE & 40 & $2.8 \pm 0.4$ & $0.067 \pm 0.014$ & $0.27 \pm 0.02$ \\
\hline M-RDE & 80 & $3.4 \pm 0.3^{\pi}$ & $0.077 \pm 0.012^{\pi}$ & $0.23 \pm 0.02^{\pi}$ \\
\hline $\mathrm{H}-\mathrm{RDE}$ & 160 & $3.8 \pm 0.2^{\pi}$ & $0.083 \pm 0.011^{\pi}$ & $0.19 \pm 0.01^{\pi}$ \\
\hline
\end{tabular}

${ }^{*} P<0.05$ and ${ }^{* *} p<0.01$ versus OVX group. L-RDE: low dose of RDE, M-RDE: middle dose of RDE, H-RDE: high dose of RDE

Table 4: Effect of RDE on biochemical parameters in the serum and urine $(n=10)$

\begin{tabular}{lccccc}
\hline Group & Dosage $(\mathbf{m g} / \mathbf{k g})$ & U-Ca/Cr(mmol/mmol) & U-P/Cr(mmol/mmol) & ALP(U/L) & OC(mmol/L) \\
\hline Control & - & $0.22 \pm 0.02$ & $3.8 \pm 0.3^{*}$ & $112.5 \pm 11.1^{*}$ & $8.1 \pm 0.2^{*}$ \\
OVX & - & $0.45 \pm 0.04$ & $5.6 \pm 0.3$ & $227.3 \pm 28.6$ & $12.3 \pm 0.4$ \\
E2 & 0.025 & $0.26 \pm 0.02^{*}$ & $4.1 \pm 0.2^{*}$ & $140.1 \pm 19.7^{*}$ & $9.2 \pm 0.3^{*}$ \\
L-RDE & 40 & $0.38 \pm 0.0 .02^{*}$ & $5.2 \pm 0.5$ & $163.4 \pm 15.8^{*}$ & $10.8 \pm 0.4$ \\
M-RDE & 80 & $0.34 \pm 0.01^{*}$ & $4.5 \pm 0.4^{*}$ & $156.7 \pm 14.9^{*}$ & $10.2 \pm 0.2^{*}$ \\
H-RDE & 160 & $0.29 \pm 0.0 .02^{*}$ & $4.3 \pm 0.3^{*}$ & $145.4 \pm 16.1^{*}$ & $9.6 \pm 0.2^{*}$ \\
\hline${ }^{*} P<0.05$ and ${ }^{* *} p<0.01$ versus OVX group. L-RDE: low dose of RDE, M-RDE: middle dose of RDE, H-RDE: high \\
dose of RDE
\end{tabular}


Decreased BMD is one of the major factors jeopardizing bone strength, resulting in increased susceptibility to fractures [26]. Thus, BMD measurement can best predict fracture risk [27]. The results in the present study showed that OVX reduced BMD in the right femurs and $L 4$ vertebrae, which are rich in trabecular bone, while treatment with RDE dose-dependently prevented the decreases in BMD. Although BMD is among the strongest predictors of facture resistance, both empirical observations and theoretical analyses show that the biomechanical properties of bone and trabecular microarchitecture influence trabecular bone strength as well [28-30]. Three-point bending tests of the left femurs in our study indicated that the higher croc in doses ( 80 or $160 \mathrm{mg} / \mathrm{kg} /$ day) prevented the OVX-induced tendency toward decreased biomechanical parameters.

Moreover, measurements of structural parameters using micro-CT also showed that treatment with RDE effectively restored the trabecular micro architectural properties compared to the OVX group. In addition, measurement of bone markers plays a role in osteoporosis diagnosis and treatment [31]. Bone mass loss, as evidenced by enhanced levels of ALP, OC, U-Ca/Cr, and $\mathrm{U}-\mathrm{P} / \mathrm{Cr}$, indicated upregulation of bone turnover by OVX. The bone turnover markers above were dose-dependently reversed by $R D E$, indicating a reduction in bone turnover rate after treatment of RDE. Our results suggested RDE's effectiveness in treating osteoporosis in menopausal women.

\section{CONCLUSION}

This study demonstrate that daily oral administration of RDE over a 12-week period prevents estrogen deficiency-induced bone loss, inhibits the deterioration of trabecular microarchitecture, maintains the biomechanical competence of bone, decreases bone turnover rate, and does not stimulate an unwanted proliferation of the uterine tissues. Our findings indicate that RDE has the potential to be further developed as a natural alternative for postmenopausal osteoporosis management in elderly women.

\section{DECLARATIONS}

\section{Conflict of Interest}

No conflict of interest associated with this work.

\section{Contribution of Authors}

The authors declare that this work was done by the authors named in this article and all liabilities pertaining to claims relating to the content of this article will be borne by them.

\section{REFERENCES}

1. NIH Consensus Development Panel on Osteoporosis Prevention, Diagnosis, and erapy. Osteoporosis prevention, diagnosis, and therapy. $J$ Am Med Ass 2001; 285: 785-795.

2. Eastell R, Black DM, Boonen S. Effect of once-yearly zoledronic acid five milligrams on fracture risk and change in femoral neck bone mineral density. J Clinical Endocr. Metabolism 2009; 94: 3215-3225.

3. Burge $R$, Dawson-Hughes $B$, Solomon $D H$. Incidence and economic burden of osteoporosis-related fractures in the United States 2005-2025. J Bone Min Res 2007; 22: 465-475.

4. Omsland TK, Emaus N, Tell GS. Mortality following the first hip fracture in Norwegian women and men. A NOREPOS study. Bone 2014; 63: 81-86.

5. Marcus R. An expanded overview of postmenopausal osteoporosis. J Mus. Neuronal Inte 2002; 2: 195-197.

6. Sugerman DT. JAMA patient page. Osteoporosis JAMA 2014; 311: 104-105.

7. Johnell O, Kanis JA. An estimate of the worldwide prevalence and disability associated with osteoporotic fractures. Osteoporosis Inter 2006; 17: 1726-1733.

8. Stevenson JC. Justi cation for the use of HRT in the longterm prevention of osteoporosis. Maturitas 2005; 51: 113-126.

9. Prelevic GM, Kocjan T, Markou A. Hormone replacement therapy in postmenopausal women. Minerva Endocrinologica 2005; 30: 27-36.

10. Gray S. Breast cancer and hormone-replacement therapy: the Million Women Study. Lancet 2003; 362: 1332-1333.

11. Orija IB, Mehta A. Hormone replacement therapy: current controversies. Clinical Endocrinol 2003; 59: 657-658.

12. Lacey JV, Mink PJ, Lubin JH. Menopausal hormone replacement therapy and risk of ovarian cancer. J Am Med Assoc 2002; 288: 334-341.

13. Rossouw JE, Anderson GL, Prentice RL. Risks and benefits of estrogen plus progestin in healthy postmenopausal women: principal results from the Women's Health Initiative randomized controlled trial. $J$ Am Med Assoc 2002; 288: 321-333.

14. Lee JK, Kim KW, Choi JY. Bisphosphonates-related osteonecrosis of the jaw in Korea: a preliminary report. $J$ Korean Assoc Oral Maxill Surgeons 2013; 39: 9-13.

15. Riggs BL, Hodgson SF, O'Fallon MW. Effect of fluoride treatment on the fracture rate in postmenopausal women with osteoporosis. New England J Med 1990; 322: 802-809. 
16. Du SH, Meng Z. The treatment of Rhizoma Drynariae for nephrasthenia syndrome and headache. J Trad Chi Med 2004; 45: 250.

17. Deng WM, Shao Y, Zhang JY. Effect of Bushen Zhuanggu Granule on relieving pain in menopausal osteoporosis patients. J Guangzhou Uni of Trad ChiMed 2007; 24: 355-358.

18. Zhang D, Liu Z, Li FM, Xie YJ. The effects of Gushudan against osteoporosis. Chi Tra Herbal Drugs 2008; 39 . 1205-1207.

19. Wong RW, Rabie B, Bendeus M, Hägg U. The effects of Rhizoma Curculiginis and Rhizoma Drynariae extracts on bones. Chi Med 2007; 2: 13-14.

20. European Commission [homepage on the internet]. Directive 2010/63/EU on the protection of animals used for scientific purposes [cited 2013 Jan 16]. Available from:

http://ec.europa.eu/environment/chemicals/lab_animals/l egislation_en.htm.

21. Wronski TJ, Dann LM, Scott KS. Endocrine and pharmacological suppressors of bone turnover protect against osteopenia in ovariectomized rats. Endocrinology 1989; 125: 810-816.

22. Turner RT, Vandersteenhoven JJ, Bell NH. The effects of ovariectomy and 17 beta-estradiol on cortical bone histomorphometry in growing rats. J Bone Min Res 1987; 2: 115-122.
23. Liu XQ, Cui L, Wu T. Study of bone histomorphometric changes at regular intervals in OVX rats. Chi J Oste 2005; 11: 427-429.

24. McElroy JF, Wade GN. Short- and long-term effects of ovariectomy on food intake, body weight, carcass composition, and brown adipose tissue in rats. Phys Beha 1987; 39: 361-365.

25. Dang ZC, Van Bezooijen RL, Karperien M. Exposure of KS483 cells to estrogen enhances osteogenesis and inhibits adipogenesis. J Bone Min Res 2002; 17: 394405.

26. Park JA, Ha SK, Kang TH. Protective effect of apigenin on ovariectomy-induced bone loss in rats. Life Sci 2008; 82: 1217-1223.

27. Cummings $S R$, Bates $D$, Black DM. Clinical use of bone densitometry: scientific review. J Amer Med Asso 2002; 288: 1889-1897.

28. Rice JC, Cowin SC, Bowman JA. On the dependence of the elasticity and strength of cancellous bone on apparent density. J Bio 1988; 21: 155-168.

29. Keaveny TM, Morgan EF, Niebur GL. Biomechanics of trabecular bone. An Rev Bio Engi 2001; 3: 307-333.

30. Allende-Vigo MZ. The use of biochemical markers of bone turnover in osteoporosis. Puer Rico Health Sci J 2007; 26: 91-95. 